\title{
Does time of day matter in clinical practice?
}

n Cite as: CMAJ 2019 September 23;191:E1063. doi: 10.1503/cmaj.1095777

Posted on cmajnews.com on Sept. 5, 2019

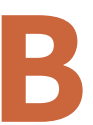

usiness experts warn against making important decisions late in the day. Judges are harsher at that time, too. But does the same hold true in medicine? There's increasing evidence that doctors may be just as prone to afternoon slumps as the next person. How to tackle the problem is less clear.

A study published in JAMA Open this year found that doctors at primary care practices in New Jersey and Pennsylvania were less likely to order cancer screening tests for eligible patients later in the day. Order rates for mammography dropped by about $16 \%$ and order rates for several colorectal cancer tests fell by about $14 \%$ from the start to the end of the day.

Other studies have shown that doctors are more prone to inappropriate prescribing of antibiotics and opioids, and give fewer flu shots later in the day. These studies and the JAMA Open paper all noted a bump in performance around lunch, followed by a slump.

These findings are typically attributed to "decision fatigue" - that is, "getting fatigued by the number of decisions you have to make," explained David Liss, a scientist at Northwestern University Feinberg School of Medicine in Chicago. "If you can limit the number of decisions people are making, there's a potential solution there."

Researchers in Philadelphia tested this idea by "nudging" doctors toward giving flu shots. In test practices, assistants recording vital signs got an electronic reminder to order the flu vaccine for eligible patients, so the order was already on the chart for approval when the primary care provider saw the patient. The overall rate of flu vaccination increased, but even with the nudge, the rate of patients getting the shot still dropped by the end of the day.

The value of prompts in the electronic medical record is limited, according to Dr. Ann-Christine Nyquist, an infection

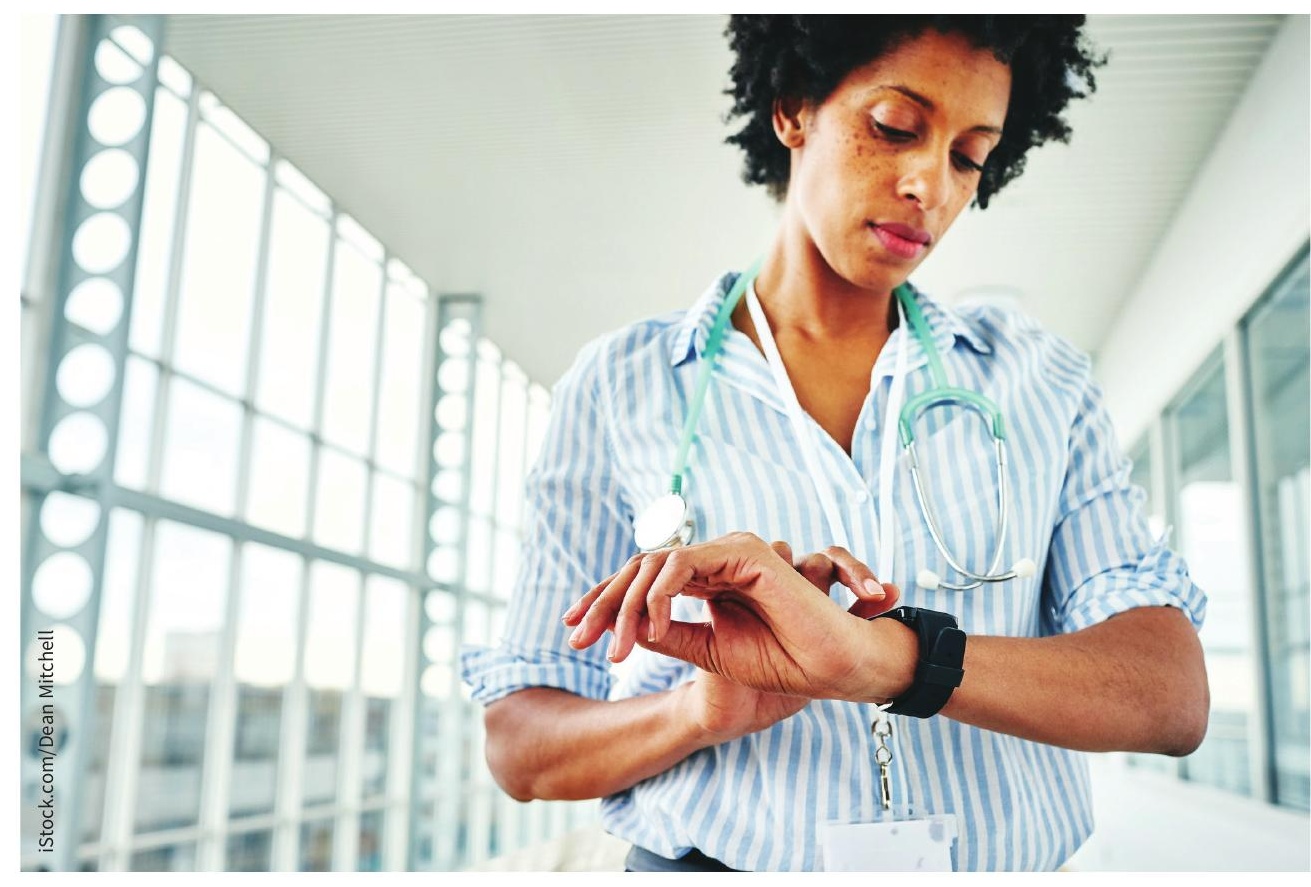

Doctors in busy primary care clinics are less thorough as the day wears on, according to recent research.

prevention specialist at Children's Hospital Colorado in Aurora. Doctors are already tired of being on the computer all the time, she said. "Something pops up again and you're like, 'Oh really? What do I have to do to ignore it?'" This can contribute to a culture of putting off flu vaccination, despite all the nudges and algorithms aimed at getting it done. "We still have the human factor making the decision and not vaccinating," Nyquist said.

Patient factors may be at play, too. The JAMA Open study found no observable differences between patients who visited the doctor at different times of day. However, the authors noted, "it could be the case that patients who see their clinician later in the day want to leave sooner and decline a discussion about cancer screening."

And in some areas of medical practice, decision fatigue doesn't seem to be an issue. In a 2016 study, Liss found that doctors performing colonoscopies were not more likely to miss findings such as adenomas of the colon, even as they faced increasing delays and it got later in the day.

Toronto surgeons who spoke to CMAJ said that time of day also tends not to affect surgical care. Dr. Bheeshma Ravi, an orthopedic surgeon who studies quality of care in the operating room, tries to schedule easier procedures earlier in the day to "warm up" for difficult cases. "It's not just for me - my team needs to warm up too," he said. Endocrine surgeon Dr. Karen Devon said she sometimes rearranges her schedule to ensure that a certain doctor is available to assist, but not to move a procedure to an earlier timeslot.

According to Liss, quality tools that are already in place may help with decision fatigue. "If there's a checklist for a particular surgery and it's standardized, then there are fewer decisions that have to be made."

Dr. Miriam Shuchman, Toronto, Ont. 